\title{
Scaling and dynamics of washboard road
}

\author{
Anne-Florence Bitbol ${ }^{1,2}$, Nicolas Taberlet ${ }^{1}$, Stephen W. Morris ${ }^{3}$, Jim N. McElwaine ${ }^{2}$ \\ 1 Université de Lyon, École Normale Supérieure de Lyon, \\ Laboratoire de Physique, 46 allée d'Italie, 69007 Lyon, France \\ ${ }^{2}$ DAMTP, University of Cambridge, Wilberforce Rd., CB3 0WA Cambridge, U.K. \\ ${ }^{3}$ Department of Physics, University of Toronto, 60 St. George St., Toronto, Ontario, Canada, M5S $1 A 7$
}

(Dated: October 24, 2018)

\begin{abstract}
Granular surfaces subjected to forces due to rolling wheels develop ripples above a critical speed. The resulting pattern, known as washboard or corrugated road, is common on dry, unpaved roads. We investigated this phenomenon theoretically and experimentally, using laboratory-scale apparatus and beds of dry sand. A thick layer of sand on a circular track was forced by a rolling wheel on an arm whose weight and moment of inertia could be varied. We compared the ripples made by the rolling wheel to those made using a simple inclined plow blade. We investigated the dependence of the critical speed on various parameters, and describe a scaling argument which leads to a dimensionless ratio, analogous to the hydrodynamic Froude number, which controls the instability. This represents the crossover between conservative, dynamic forces and dissipative, static forces. Above onset, wheel-driven ripples move in the direction of motion of the wheel, but plow-driven ripples move in the reverse direction for a narrow range of Froude numbers.
\end{abstract}

PACS numbers: 45.70.Qj,45.70.-n

The spontaneous rippling of gravel roads after the passage of many vehicles is annoyingly familiar to drivers of unpaved roads around the world. Avoiding or mitigating the rippling effect, known as washboard or corrugated road, is a significant engineering challenge [1, 2, 3, 4, 5. 6, 7]. We have argued recently [8] that the rippling can be regarded as a type of nonlinear pattern-forming instability [9] of the flat road [10, 11]. This nonlinear physics point of view is in many ways complementary to the engineering one, and leads to new insights. We present here an experimental and theoretical study of the region near the onset of the instability, which appears only above a critical threshold speed. We focus on how this critical speed scales with various parameters. We also survey some of the complex dynamics that occurs above onset [10].

Washboard road is commonly, and incorrectly, assumed to require the forced oscillation of the suspension of the vehicle 77. Although a suspension system does change the quantitative details, ripple frequencies are generally quite far from the free resonant frequencies of the suspension [2, 7], and ripples can appear even with no suspension present [8]. Here, we consider a simplified experimental system in which a wheel merely returns to the road surface under gravity. We also study the even simpler case of a non-rotating, angled "plow" blade in place of the rolling wheel. In both cases, well above onset, we find that the ripples travel down the road in the direction of driving and that the wheel or plow is thrown free of the roadbed between ripples. In the case of the plow, there also exists a range just above onset where the blade remains in contact with the surface and the ripples travel in the reverse direction, against the direction of driving.

The rippling instability is quite robust, and has analogs in other similar effects on granular surfaces, such as wind 12 and water-driven 13 ripple patterns. Other related phenomena are, for example, periodic wear patterns on railroad tracks [14, mogul formation on ski slopes [15, skipping stones over water [16] and certain failure modes of computer hard disks [17, 18. What all these phenomena have in common is a repeated, nonlinear interaction between a moving source of lateral and vertical contact stresses acting on a deformable or erodible surface. The interaction includes the case when the source of stress, which both deforms and responds to the shape of the surface, completely leaves the surface so that the stresses go to zero. We propose a very general picture of the key mechanism of the washboard instability in terms of a dimensionless group, analogous to the hydrodynamic Froude number, which we show controls the scaling of the onset speed. This analysis may be broadly generalizable to the diverse situations cited above.

Previous experimental work and soft sphere molecular dynamics simulations 8 have shown that neither compaction nor size segregation processes in the granular bed are essential for the instability, contrary to some theories [11. Engineering studies [1, 2, 3, 4, 5, 6] have mainly concentrated on optimizing the response of a suspension system to fully developed ripples and have not typically considered the threshold of the instability. Using our approach, it may be possible to suppress or delay the onset of this threshold by using appropriate control strategies based on the nonlinear dynamics of the coupled wheelroadbed system, which behaves in some respects like an impact oscillator [19, 20].

This paper is organized as follows; in the section [. we describe the experimental apparatus. This is followed in section [II by a qualitative discussion of the rippling phenomena that are observed for both wheels and plow blades. In section III, we consider the theory of the basic scaling of the onset speed, which we then examine exper- 

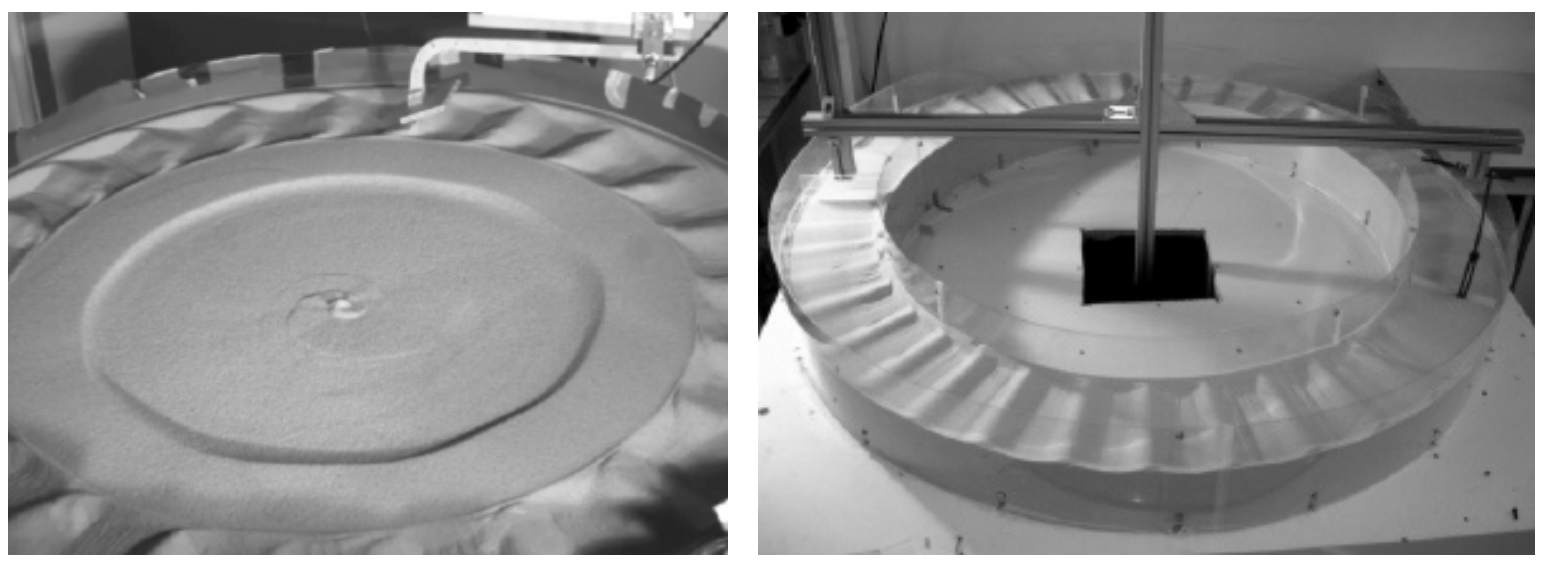

FIG. 1: The two versions of the experimental apparatus. The left photo shows the setup in Cambridge, where the table carrying the sand roadbed rotates beneath an arm holding a wheel or plow, which is stationary in the lab frame. The right photo shows the setup in Lyon, where the roadbed is stationary beneath an arm that rotates. In both experiments the moving parts rotate counterclockwise, as seen from above.

imentally in section [V] Next, in section $\mathrm{V}$ we discuss the regime of backward traveling ripples and in section VI we describe some of the rich variety of ripple states that appear above onset. Finally, section VII is a brief conclusion.

\section{EXPERIMENTAL METHOD}

Two versions of the experimental apparatus were used in this study, as shown in Fig. 1. The first was essentially that of Ref. [8] and consisted of a $1 \mathrm{~m}$ diameter rotating table, with a maximum rotation speed of $0.8 \mathrm{~Hz}$, holding a $200 \mathrm{~mm}$ deep layer of rough sand, producing a moving roadbed. This passed under an arm which could hold either a rolling wheel or a plow blade which was stationary in the lab frame. This arrangement has some advantages for visualization and also the large moment of inertia of the table helps maintain a constant speed. In the second version of the apparatus, the arm holding the wheel or plow hangs from a rotating axle over a $1.80 \mathrm{~m}$ diameter roadbed which is stationary in the lab frame. This version is larger and can reach higher speeds, with a maximum axle rotation frequency up to $1 \mathrm{~Hz}$. In both versions, we used rough sand with a grain diameter of $300 \pm 100 \mu \mathrm{m}$. We have previously shown [8] that the shape and size of the grains is unimportant to the rippling phenomenon. The density of the grains, which was not varied in the present study, is however a crucial parameter, as discussed in section III below.

Fig. 2 shows the arm with the wheel and plow blade configuration in detail. A light, $330 \mathrm{~mm}$ long arm pivoted around a point of support. An optional counterweight could be added to the end opposite the wheel or plow. The weight and transverse width of the wheel or plow can be varied, and, in the case of the plow, the angle of attack $\alpha$ was also adjustable. The wheel used was smaller and lighter than that described in Ref. [8, having a diameter of $65 \mathrm{~mm}$ and a typical width of $25 \mathrm{~mm}$. The plow blade was typically $120 \mathrm{~mm}$ wide and inclined at an angle of $\alpha=40^{\circ}$.

The masses of the wheel or plow and counterweight could be varied between $0.045 \mathrm{~kg}$ and $3 \mathrm{~kg}$. As discussed in section III below, these arrangements are dynamically equivalent to a physical pendulum, as shown in Fig. 2.2. Since the angle of the arm $\theta$ was small, the physical pendulum was always nearly horizontal. Thus, the wheel or plow behaved as if it were a freely falling object and the natural frequency of the pendulum played no role. No spring or dashpot was used, so this arrangement constitutes a minimal suspension having no resonant or natural frequencies, and only rather weak damping.

The wheel rolled freely and no torques were applied to it other than that produced by contact with the surface. The plow was simply dragged over the surface. The speeds were inferred from measurements of the rotation rate of the table and spanned the range $0-6 \mathrm{~ms}^{-1}$. A magnetic angle sensor [21] was attached to the pivot supporting the arm, and its angle $\theta$ was digitized and used to find the vertical position $z$ of the wheel or plow blade. $z$ was measured with a vertical accuracy of $0.2 \mathrm{~mm}$ at a rate of $2000 \mathrm{~Hz}$. The position data was acquired continuously along with data from an optical encoder that generated a pulse each table rotation.

The dynamics of the ripple pattern evolved rather slowly, especially near the critical speed $v_{c}$ for the onset of the instability. It was thus necessary to increase and decrease the speed over periods of many hours to achieve quasi-static, steady state patterns. In some runs, the speed was ramped up quickly from a standing start, beginning with a flat sand bed. This protocol is analogous to making a rapid "quench" into the rippled state. In other runs, a small perturbation was made in the bed to act as a nucleation site for ripples. 

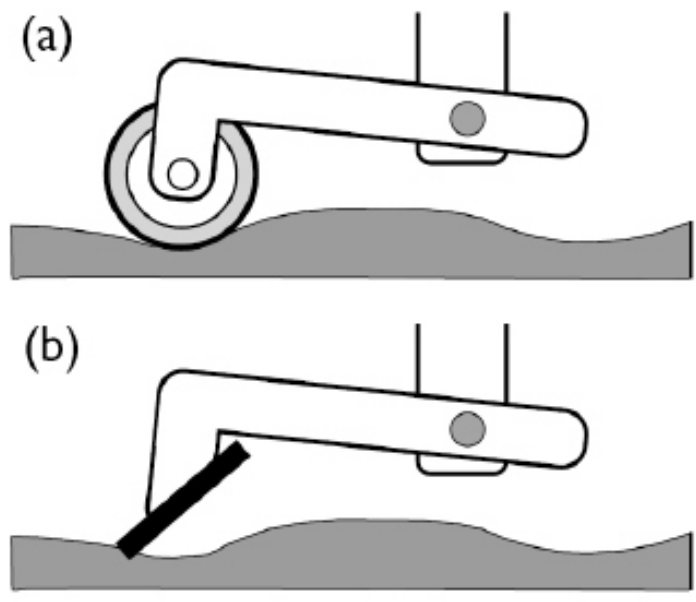

(c)

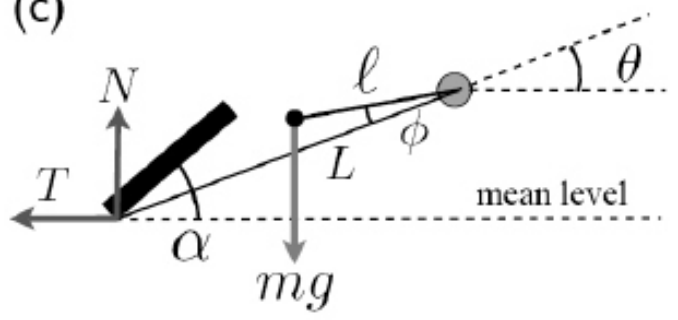

FIG. 2: Schematic views of (a) the wheel and (b) plow blade. (c) shows the geometry of the equivalent physical pendulum. The plow is rigidly fixed to the arm and makes an angle of attack $\alpha$ with respect to the horizontal.

\section{QUALITATIVE RESULTS}

In this section, we discuss some of the phenomena observed, before focusing on the scaling of the critical speed for the onset of rippling in section [V] Initially, we tried preparing the roadbed by running a comb through it to make a loose, flat, granular assembly. However, if the experiment is run continuously over a period of around a week ( $\approx 100000$ rotations), the bed gradually compacts and the thresholds and amplitudes slowly change. We therefore used a different protocol where the sand was disturbed as little a possible. To prepare a flat bed, the experiment was run at a speed below the onset for many rotations. The results were then repeatable.

Ripples driven by either the wheel or the plow only appear above a well-defined critical speed $v_{c}$. Below this speed, perturbations made in the roadbed are observed to be smoothed away by the action of the wheel or plow. For speeds near $v_{c}$, the growth rate of perturbations is nearly zero, and it can take many thousands of rotations of the experiment before ripples build up. The quasi-steady state ripples which develop after the initial transient have a very small amplitude just above $v_{c}$. For all cases, speeds well above $v_{c}$ result in regimes where the wheel or plow is thrown completely off the roadbed near the crest of a ripple, before landing again on the front face of the next
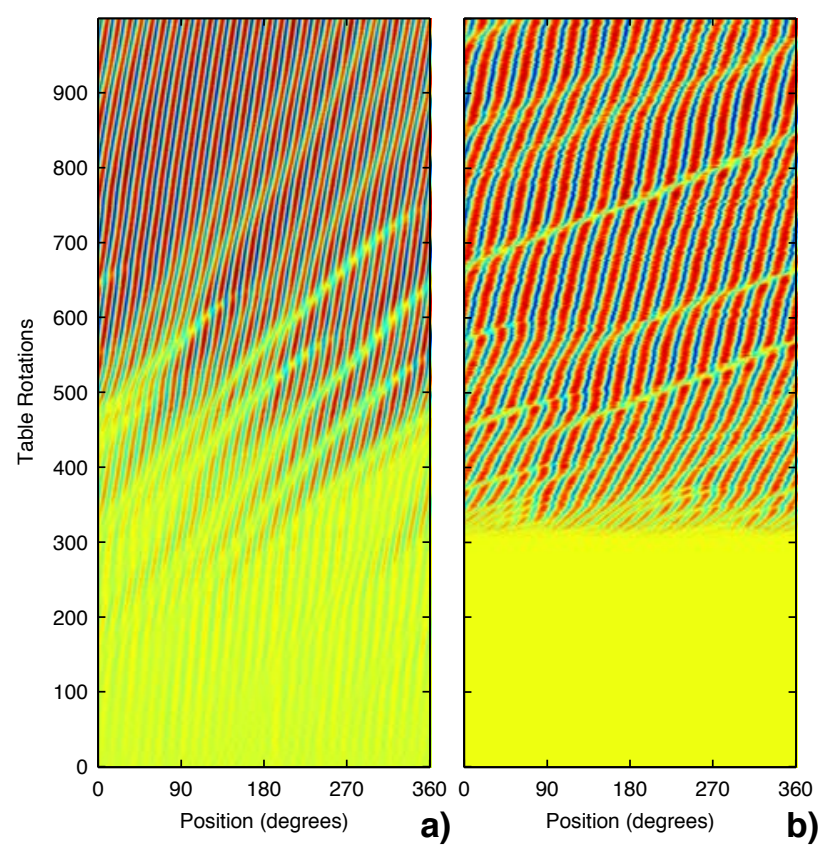

FIG. 3: (Color online) Typical space-time plots showing the development of ripples from a flat bed, with red indicating a higher arm position and blue a lower. Both parts show 1000 rotations and have the same scale. The driving direction is to the right. a) A free wheel just above the critical velocity. b) The plow, with $v$ below the critical velocity for the first 300 rotations and well above critical for the next 700 rotations.

ripple. The resulting large amplitude ripples are strongly asymmetric, with a gradual rise on their front faces, and an abrupt slip-face on the back side. Nearer to $v_{c}$, the ripples are more symmetrical.

The plow is observed to remain in contact with the surface at all times near $v_{c}$. The ripples move in the direction of driving for wheels, but may move in the reverse direction for plows, if $v$ is close to critical. At larger speeds, ripples move in the direction of driving in both cases. We discuss this curious reversing behavior in section $\mathrm{V}$ below. Fig. 3 shows some representative space-time plots of ripple evolution after a quench into the strongly unstable regime. During the transient, numerous ripple creation and annihilation events are observed, resulting in adjustments of the pattern which tend to propagate in the direction of driving. Fig. 4 4 shows a quench close to the threshold with the plow showing backward ripples. Eventually, a quasi-steady state of traveling ripples is established. For sufficiently deep quenches, the pattern can display multi-modal behavior or persistent time dependence, as discussed in section $\mathrm{V}$. The final, saturated amplitude of the ripples increases with the driving speed near the threshold, but shows non-monotonic behaviour far above.

It is easy to observe experimentally that $v_{c}$ depends on the mass of the wheel or plow, and the moment of inertia of the arm. By changing the granular material, it was apparent $[8$ that only the density of the roadbed 


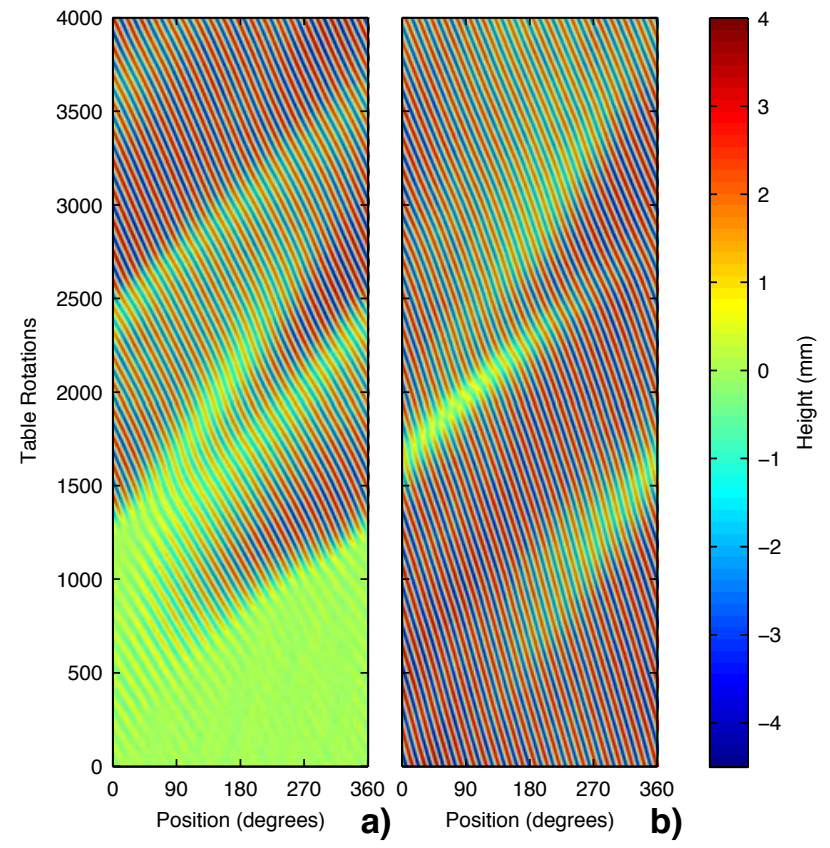

FIG. 4: (Color online) Parts a and b show different segments of a ten day experiment during which the speed was decreased in steps of $0.0021 \mathrm{~m} \mathrm{~s}^{-1}$ every 500 rotations. The driving direction is to the right. In part a, the speed is increasing from $0.684 \mathrm{~m} \mathrm{~s}^{-1}$ to $0.691 \mathrm{~m} \mathrm{~s}^{-1}$ (Fr 2.04-2.09), triggering a transition from flat bed to backward traveling ripples. In $\mathrm{b}$, The speed is decreasing from $0.694 \mathrm{~m} \mathrm{~s}^{-1}$ to $0.687 \mathrm{~m} \mathrm{~s}^{-1}$ (Fr 2.10-2.06), triggering a transition from 21 to 20 ripples. Here $M_{G}=0.0785 \mathrm{~kg}, w=0.120 \mathrm{~m}, \rho=1201 \mathrm{~kg} \mathrm{~m}^{3}$, and the angle of attack $\alpha$ was $36.8^{\circ}$

material matters, and not the size or shape of the grains. These dependencies can be accounted for by the scaling analysis discussed in the next section.

\section{SCALING THEORY}

In this section, we consider the dynamics of the arm assembly and its interaction with the sand bed. We seek to identify the essential physical parameters that govern the scaling of the critical onset speed $v_{c}$. To simplify the problem, we consider a strictly two-dimensional model in which the transverse width of the wheel or plow is assumed to be large compared to its penetration into the bed. This assumption was exactly true for the soft sphere molecular dynamics simulations discussed in Ref. 8], which qualitatively reproduced all of the essential features of the experiment. The validity of this simplifying assumption for the real experiment will be discussed in section IV.

The geometry of the arm and plow assembly is shown in Fig. 2k. The position of the assembly is completely specified by three points: the contact point with the bed, the pivot position and the location of the center of mass.
These three positions define two intrinsic lengths, the distance from the pivot point to the center of mass $\ell$, and the distance from the pivot point to the contact point with the bed $L$. The angle $\phi$ between these lines is constant. The angle $\alpha-\theta$ between the plow and the line to the pivot point is also fixed, whereas $\theta$ will vary as the bed deforms.

Dynamically, the system can be described as a forced physical pendulum of length $\ell$, mass $m$ and moment of inertia about the center of mass $I$. The latter two quantities define another length $\sqrt{I / m}$. There is one additional length, the transverse width of the plow or wheel $w$. Thus, to describe the whole assembly requires three angles, $\alpha, \theta$ and $\phi$ (which are of course non-dimensional), four lengths $\ell, L, \sqrt{I / m}$ and $w$, the mass $m$ and the acceleration due to gravity $g$. The properties of the bed involve only one additional parameter, the sand density $\rho$, as discussed below.

The gravitational force $m g$ acts through the center of mass and exerts a torque $m g \ell \cos (\theta-\phi)$ about the pivot point. The force of the bed on the arm is assumed to act entirely at the contact point. It exerts a torque about the pivot point of $-L(N \cos \theta+T \sin \theta)$, where $N$ is the vertical (normal) force from the bed and $T$ the horizontal (tangential) force. Since the moment of inertia about the pivot point is $I+m \ell^{2}$, conservation of angular momentum gives

$$
\left(I+m \ell^{2}\right) \ddot{\theta}=m g \ell \cos (\phi-\theta)-L(N \cos \theta+T \sin \theta) .
$$

The vertical height of the contact point relative to the pivot point is $z=-L \sin \theta$. To determine how the onset speed $v_{c}$ scales, we consider small displacements about a flat bed, so that $\theta$ is small. This is sufficient, since the transition to rippling occurs at zero amplitude. We treat $\theta$ as constant except in the $\ddot{\theta}$ term where $\ddot{z}=-L \ddot{\theta}$. After dividing Eqn. 1 by $L$ and substituting for $z$ we get approximately

$$
\frac{M_{I}}{\cos \theta} \ddot{z}=(N \cos \theta+T \sin \theta)-M_{G} g \cos (\theta-\phi),
$$

where $M_{I}=\left(I+m \ell^{2}\right) / L^{2}$ is the effective inertial mass and $M_{G}=m \ell / L$ is the effective gravitational mass.

$M_{I}$ and $M_{G}$ can be changed independently by adjusting $\ell, L$ and $m$. They are equal if all the mass is located at the end of the arm. If the angular dependencies are absorbed into the definitions, that is with $M_{I}^{\prime}=M_{I} / \cos \theta$, $M_{G}^{\prime}=\cos (\theta-\phi) M_{G}$, and $N^{\prime}=N \cos \theta+T \sin \theta$, then for small displacements (i.e. small changes in $\theta$ ) we have the equation of motion

$$
M_{I}^{\prime} \ddot{z}=N^{\prime}-M_{G}^{\prime} g,
$$

which is simply that of a falling mass interacting with a surface via a normal force $N^{\prime}$. This equation holds for small $\theta$, but for larger $\theta$, the coupling of the resistive force $T$ to the vertical motion becomes important. Our experiments concentrated on small $\theta$, but in subsection IVC, 
we discuss the results of experiments where this condition is relaxed.

The mechanics of the granular road bed are not so straightforward to model as those of the arm. We aim for a minimal description that leads to the correct scaling of the fully coupled problem. Much more detailed analyzes of the rheology of the granular material would be required for a very realistic description [3].

We consider the case where the depth of penetration into the bed is small compared to the width of the object, so that we may assume that the induced flow in the sand will be essentially two-dimensional, and that edge effects are small. This assumption is probably violated in our experiments with the narrowest wheels and plows.

The sand grains were dry and sufficiently large so that they were non-cohesive. Previous work [8] has shown that changing the grain size does not change the wavelength, amplitude or critical velocity of the ripples. Therefore, the granular medium can be described by only its threedimensional density $\rho$ and possibly by some other nondimensional parameters. The shallowness assumption and two-dimensionality means that the density $\rho$ of the sand and the width $w$ will only occur in the form of a two-dimensional density $\rho w$, and that the width should have no further role. It may be possible to allow for some three dimensional effects by allowing a virtual origin correction in the width, though we have not tested this.

To arrive at a plausible scaling, we examine the balance of forces for a plow or wheel moving over and penetrating the sand surface. Forces between the bed and the plow or wheel arise in two distinct ways which scale differently with $v$. One of these is almost entirely dissipative and the other one is almost entirely conservative. We postulate that the onset of washboard ripples occurs when the relative sizes of these forces cross over as a function of $v$. We consider the geometrically simpler case of the plow first.

Static, buoyancy or frictional forces must be proportional to $A \rho w g$, where $A$ is the displaced area of granular material. For a plow penetrating a distance $h=z_{0}-z$, where $z_{0}$ is the free surface position, they will be proportional to $h^{2} \rho w g$. These forces will be almost entirely dissipative. On the other hand, dynamic forces, those arising from the inertia of the sand, will be proportional to $h \rho w v^{2}$. These forces are mostly conservative. We neglect the vertical velocity $\dot{h}$ as small compared to $v$. Ignoring constants and angular dependence, we separately balance these two penetration forces against the effective weight of the arm $M_{G} g$. This gives the static and dynamic equilibrium penetration depths

$$
\begin{aligned}
& h_{s}=\sqrt{\frac{M_{G}}{\rho w}}, \\
& h_{d}=\frac{M_{G} g}{v^{2} \rho w} .
\end{aligned}
$$

As $v$ increases, the dynamic forces increase and the dynamic penetration depth $h_{d}$ needed to support the weight decreases, whereas the static forces are unaffected. Since the static forces are almost completely dissipative and the dynamic forces almost completely conservative, the switch between the two that occurs as $v$ increases explains the transition from a highly dissipative furrowplowing mode to one in which the plow spends some of the time in non-dissipative, ballistic flight. For low speeds $v$, the normal forces between the plow and the sand do only dissipated work against friction, while for larger $v$, the normal force is sufficient to throw the plow off the surface and into free flight. Ripples are then formed and maintained by the periodic dissipative collisions with the surface. The onset of washboard ripples in this picture is thus somewhat analogous to the onset of skipping for a stone projected over a water surface [16]. A key difference, of course, is that the granular ripple pattern is built up by many passes of the plow, each of which contributes only a small, but persistent, deformation of the surface. The important non-linearity in the problem is the highly nonlinear nature of the normal force, which abruptly goes to zero when the plow leaves the surface, as is the case for impact oscillators [19, 20].

The dimensionless ratio of the two penetration depths,

$$
\operatorname{Fr}=\frac{h_{s}}{h_{d}}=\frac{v^{2}}{g} \sqrt{\frac{\rho w}{M_{G}}},
$$

is analogous to the Froude number used in similar hydrodynamic arguments [16. We propose [8] that this dimensionless group controls the onset of the instability, with ripples setting in above a critical, threshold value of $\mathrm{Fr}=\mathrm{Fr}_{\mathrm{c}}$. Our scaling argument does not furnish any estimate of the value of $\mathrm{Fr}_{\mathrm{c}}$, beyond the general expectation that $\mathrm{Fr}_{\mathrm{c}} \sim O(1)$. We establish experimental values of $\mathrm{Fr}_{\mathrm{c}}$ in section IV] below. We will also show cases where the plow does not actually leave the surface, but still forms backward traveling ripples for $\mathrm{Fr} \gtrsim \mathrm{Fr}_{\mathrm{c}}$.

We have physically motivated the identification of the Froude number in terms of a crossover between dissipative and conservative forces 22, but it can also be deduced from straightforward dimensional analysis. If the only relevant dimensional quantities of the arm that determine the transition are its effective gravitational mass $M_{G}$ and its width $w$, and if we assume the twodimensional model such that $w$ and $\rho$ can only appear as the product $\rho w$, then Fr is the only dimensionless group that can be formed from a combination of $M_{G}, \rho w, v$ and $g$.

The critical Froude number $\mathrm{Fr}_{\mathrm{c}}$ will also depend on $\theta$, $\alpha, \phi$ and possibly on other dimensionless properties of the bed. If these are held constant, Eqn. 6 implies that the critical speed $v_{c}$ is given by

$$
v_{c}=\left(\operatorname{Fr}_{\mathrm{c}} g\right)^{1 / 2}\left(\frac{M_{G}}{\rho w}\right)^{1 / 4} .
$$

In subsection IVA, we will show experimentally that $v_{c}$, for the plow, does indeed exhibit an $M_{G}^{1 / 4}$ scaling. In 
Sections IVB and IVC we consider the dependence of $v_{c}$ on $w, \alpha$ and $\theta$.

The wheel is more complicated than the plow in some ways as there is an additional length scale, the wheel radius $R$. The displaced volume for a cylinder length $w$ which penetrates to depth $h$ is

$$
\begin{aligned}
& w\left[R^{2} \cos ^{-1}(1-h / R)-(R-h) \sqrt{2 R h-h^{2}}\right] \\
= & \frac{4}{3} w h \sqrt{2 R h}[1+O(h / R)] .
\end{aligned}
$$

Thus the scaling is now $A \propto h^{3 / 2}$ instead of $A \propto h^{2}$, so that the static depth is modified. For the wheel

$$
h_{s}^{\text {wheel }}=R^{-1 / 3}\left(\frac{M_{G}}{\rho w}\right)^{2 / 3} .
$$

The dynamic depth is the same thus the scaling of the Froude number is changed to

$$
\mathrm{Fr}^{\text {wheel }}=\frac{h_{s}^{\text {wheel }}}{h_{d}}=\frac{v^{2}}{g}\left(\frac{\rho w}{M_{G} R}\right)^{1 / 3}
$$

Thus, we predict the following relation for the critical speed

$$
v_{c}^{w h e e l}=\sqrt{\operatorname{Fr}_{\mathrm{c}}^{\text {wheel }}}\left(\frac{g^{3} M_{G} R}{\rho w}\right)^{1 / 6},
$$

where numerical coefficients have been ignored.

To experimentally determine the relevant parameters of the model, it suffices to determine the three constant quantities that appear in Eqn. 2, namely $M_{I}, M_{G}$ and $\phi$. We supported the arm on a balance, so that the bottom of the plow was exactly level with the pivot point and $\theta=0$. Then the force measured by the balance was $N=$ $M_{G} g \cos \phi$. The angle $\phi$ was determined by suspending the arm from the pivot point and measuring $\theta$. Then $M_{G} g \cos (\theta-\phi)=0$ and $\phi= \pm \pi / 2+\theta$. Eqn. 2 for the suspended arm is simply the equation of a free physical pendulum,

$$
M_{I} L \ddot{\theta}=M_{G} g \cos (\phi-\theta),
$$

which, for small amplitudes, oscillates with angular frequency $\omega=\sqrt{M_{G} g / M_{I} L}$. The length $L$ was directly measured with a ruler, while $\omega$ was measured by triggering small oscillations, and then measuring $\theta$ using the magnetic sensor. In this way, all the parameters in Eqn. 2 were determined. All these quantities vary slightly with the angle of attack $\alpha$ of the plow, and so were measured for a range of $\alpha$. The plows of different width were carefully chosen so as not to alter any of the properties.

\section{SCALING OF THE THRESHOLD SPEED}

To test the scaling theory outlined in the previous section, we measured the critical speed $v_{c}$ for a variety of plow and wheel configurations. The transition speed is most accurately determined by running the system at a constant speed above $v_{c}$ until a steady state has developed. The speed is then decreased by steps of $0.0016 \mathrm{~m} \mathrm{~s}^{-1}$ and kept at each speed for 1000 rotations. This is more accurate and quicker than starting with an initial perturbation or flat bed and observing the subsequent behaviour, as a very large number of rotations are necessary for the ripples to develop near $v_{c}$. We also recorded the long-term dynamical states for a range of speeds from below to well above onset.

As discussed in the following subsections, we added various masses and counterweights to the arm, which has the effect of varying $M_{I}$ and $M_{G}$, as well as varying the width of the plow $w$, its angle of attack $\alpha$ and the mean support angle $\theta$.

\section{A. Variation with gravitational mass $M_{G}$}

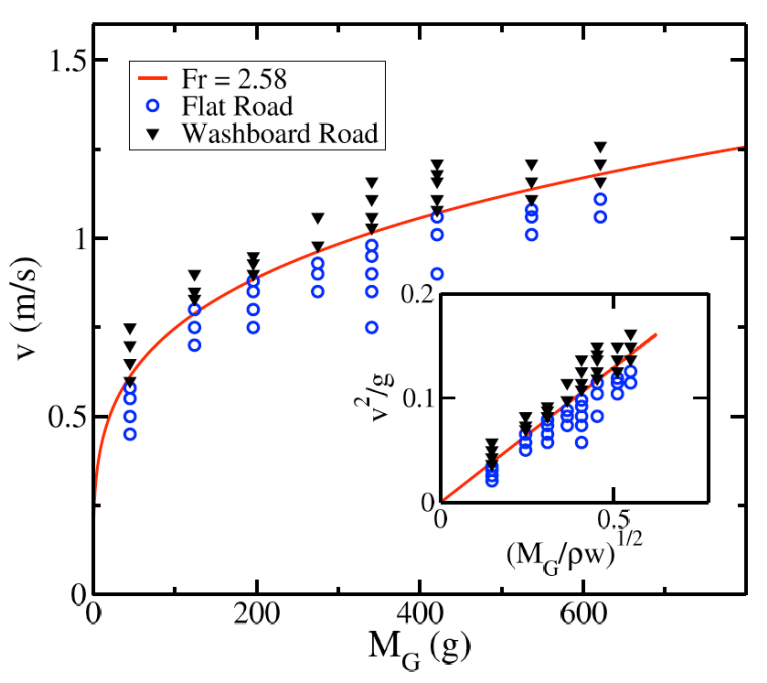

FIG. 5: (Color online) The domain of existence of the ripple pattern for the plow, as a function of the speed $v$ and the gravitational mass $M_{G}$. The solid line corresponds to a constant Froude number $\mathrm{Fr}=2.58$. The inset shows $v^{2} / g$ vs. $\left(M_{G} / \rho w\right)^{1 / 2}$, a scaling for which Eqn. 6 predicts the boundary to be a straight line.

Fig. 5 shows the variation of $v_{c}$ with the gravitational mass $M_{G}$ for the plow. The frontier between the flat bed and the appearance of washboard ripples is well described by an $M_{G}^{1 / 4}$ scaling, corresponding to a critical Froude number of $\mathrm{Fr}=2.58$. This result clearly validates the theory presented in the previous section, and indicates that the instability is controlled by Fr. It is interesting to note that the first patterns encountered above onset are backward traveling ripples for which the plow does not lift off the surface. These are discussed in more detail in section $\mathrm{V}$ below.

The scaling theory is less successful for the case of the rolling wheel; Fig. 6 shows $v_{c}$ vs. $M_{G}$ for the wheel. To 
a)

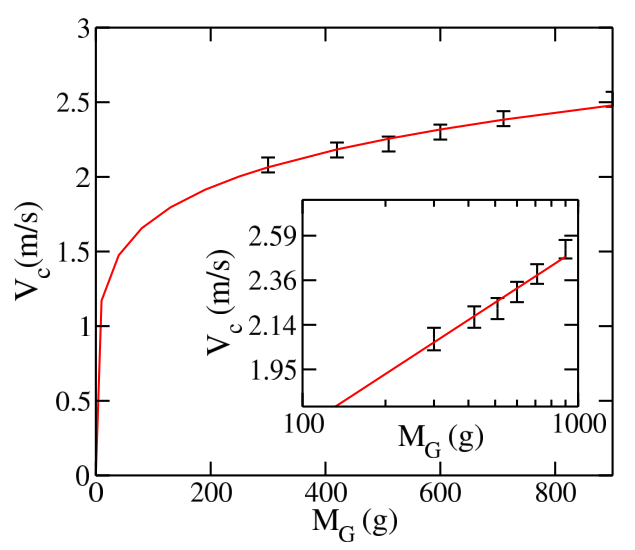

b)

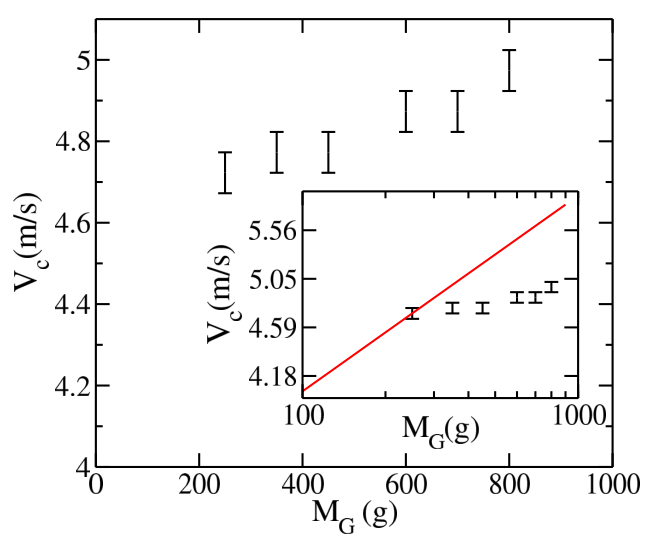

FIG. 6: (Color online) The critical speed $v_{c}$ for the onset of the ripple pattern for wheels. Part (a) shows $v_{c}$ vs. $M_{G}$ for "blocked", or non-rolling wheels, while (b) shows the same data for freely rolling wheels. The solid line corresponds to a constant Froude number, according to Eqn. 11, i.e. to an $M_{G}^{1 / 6}$ power law dependence. It is evident that the predicted scaling is not obtained for rolling wheels.

isolate the effects of rolling from the purely geometrical dependence, we measured $v_{c}$ both for wheels that were free to roll and for otherwise identical wheels that were "blocked" - i.e. prevented from rotating. A blocked wheel behaves much like a differently shaped plow.

Fig. 6 a shows that the predicted $M_{G}^{1 / 6}$ scaling is consistent with the blocked wheel data. However, experimental limitations only allowed us to vary $v_{c}$ over a range of $20 \%$, so the correctness of this scaling is not conclusively demonstrated. It is clear however that this scaling fails for the case of a rolling wheel, as shown in Fig. 6p. This is perhaps not surprising since arguments presented in section III were based only on the geometry of the wheel and completely ignored its rotation. Evidently, the rolling component of the motion affects the surface penetration properties of the wheel, invalidating the scaling based only on geometry. The contact forces for a rolling wheel, and their effect on the deformation

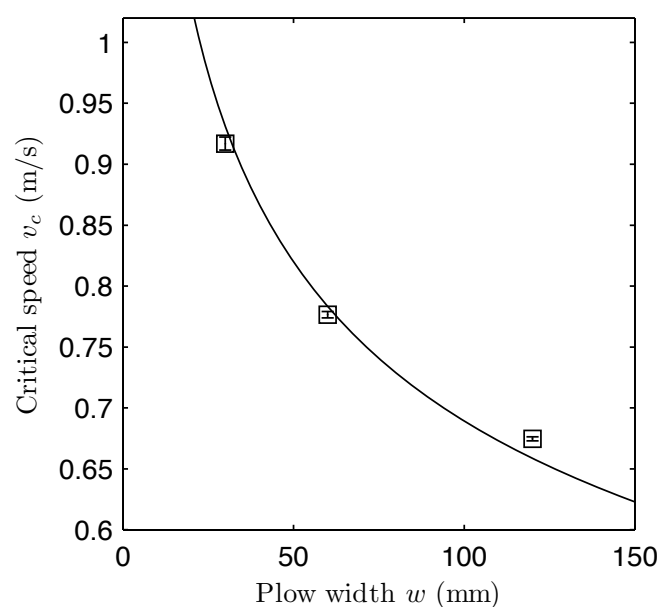

FIG. 7: The dependence of the critical velocity $v_{c}$ on the width $w$ of the plow. The Froude numbers of the three points shown were 1.84, 1.86 and 1.99, and hence the critical Froude number $\operatorname{Fr}_{\mathrm{c}}$ remained approximately constant while $w$ was varied. The solid line shows a satisfactory fit to the $w^{1 / 4}$ scaling expected from Eqn. 7.

of the granular surface, presumably scale with additional powers of $R$ and perhaps also depend on the moment of inertia of the wheel. In any case, Fig. 6 shows that the gravitational mass dependence of $v_{c}$ is extremely weak for wheels, rolling or otherwise.

\section{B. Variation with plow width $w$}

The scaling argument presented in section IV depended on the assumption that the motion was essentially two-dimensional so that only the two-dimensional density $\rho w$ mattered. This is probably the most drastic approximation in the scaling theory. Figure 7 shows how $v_{c}$ scales with $w$ for the plow, for constant $\alpha$ and $M_{G}$. The data is well fit by a $w^{-1 / 4}$ dependence, as predicted by Eqn. 7, and we do find that the critical value of $\mathrm{Fr}$ remains constant even as $w$ is varied by a factor of four. The $w$ scaling can only hold for a relatively small range of plow widths, however. For very narrow plows, $w \sim h$ and the two-dimensional scaling will break down. On the other hand, for very wide plows $w$ becomes a significant fraction of the radius of the circular bed and thus there is a significant variation of $v$ across the width. Figure 7 spans the intermediate range of $w$ where a reasonable agreement is obtained.

\section{Variation with the angles $\alpha$ and $\theta$}

The onset of the washboard ripples depended strongly on the angle of attack of the plow $\alpha$, and on the arm angle $\theta$, as defined in Fig. 2, $\alpha$ could be varied, while keeping all other parameters constant. $\theta$ depended on the 


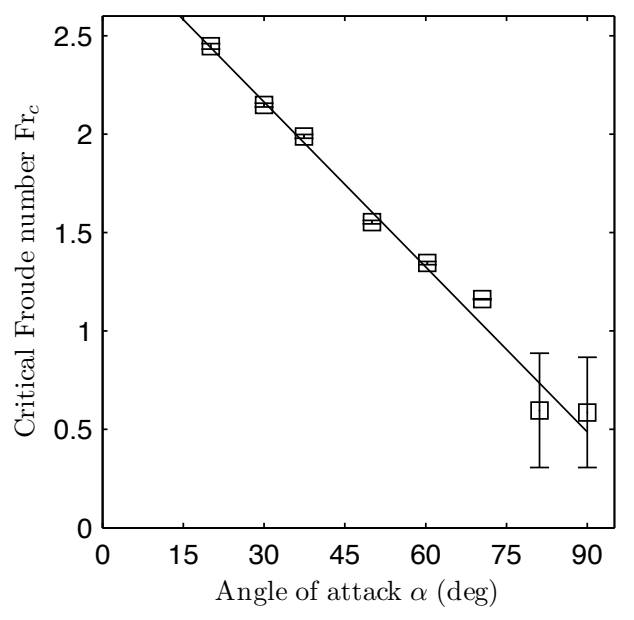

FIG. 8: The dependence of the critical Froude number on the angle of attack of the plow. The angle $\alpha$ is measured with respect to the horizontal (see Fig. 2). At low angles, the transition was very sharp and the error bars lie within the symbols.

height of the supporting structure, and could be adjusted independently of $\alpha$. Due to experimental limitations, $\theta$ could only be varied over a range of about $15^{\circ}$. The two angles were in fact somewhat interdependent, as the mean height of the bed could slowly change over time due to compaction or to sand moving to other parts of the table. In such cases, $\alpha$ and $\theta$ both drifted upward by a few degrees over the course of an experiment.

The simple scaling theory of Eqn. 7 does not explicitly predict the $\alpha$ and $\theta$ dependence of the critical speed $v_{c}$, which would be expected to show up as a variation of the critical Froude number $\mathrm{Fr}_{\mathrm{c}}$. Fig. 8 shows how $\mathrm{Fr}_{\mathrm{c}}$ depended on $\alpha$. We found that $v_{c}$, and hence $\mathrm{Fr}_{\mathrm{c}}$, strongly decreased as $\alpha$ increased. This can be explained by the observation that the transport of sand along the bed increases when $\alpha$ is increased. It is not a surprise, therefore, that the velocity needed to trigger the washboard instability should decrease with increasing $\alpha$. The case of $\alpha \approx 90^{\circ}$, a nearly vertical plow, is somewhat peculiar. At this point, the transition becomes poorly defined and the amplitude noise near onset greatly increases. At $\alpha \approx 90^{\circ}$, it appears that other parameters such as the thickness of the plow may play an important role.

The systematic interdependence of $\alpha$ and $\theta$ makes it quite difficult to exactly repeat the experimental conditions. The error bars in Fig. 8 are the precision that the transition can be identified within a particular experimental run rather than the somewhat larger error bars that would be obtained from trying to achieve exactly the same $\theta$ over many runs.

The critical speed $v_{c}$ also shows a strong dependence on $\theta$. Fig. 9 shows how the critical Froude number scales with variations in $\theta$, with $\alpha=45 \pm 3^{\circ}$. If we directly apply Eqn. 1. and make a naïve model of the friction

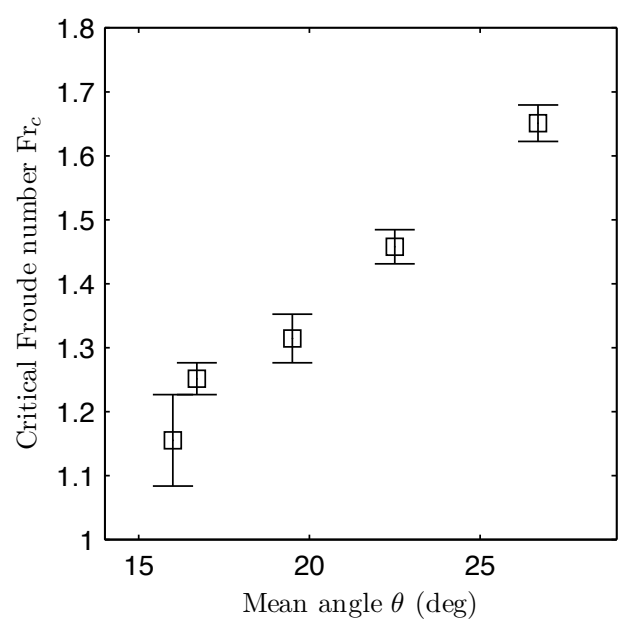

FIG. 9: The dependence of the critical Froude number on the mean angle $\theta$ that varies with the height of the support.

forces, we would guess that

$$
v_{c} \sim\left[\frac{g^{2} M_{G}}{\rho w}\right]^{1 / 4}\left(\frac{\cos (\phi-\theta)}{\cos \theta+\mu \sin \theta}\right)^{1 / 4},
$$

where $\mu$ is the unknown ratio between the normal force $N$ and the tangential force $T$. For $O(1)$ values of $\mu$ and small $\phi$, this predicts that the critical Froude number should decrease weakly with increasing $\theta$. This is the opposite of what is observed. Clearly, the $\theta$ dependence of the critical Froude number is not so easily explained and a more detailed study is required to account for it.

\section{BACKWARD-TRAVELING RIPPLES}

In all experiments using wheels, including our previous studies [8], the ripples that appear just above onset were observed to travel in the direction of driving. This agrees with most, but not all, observations in previous engineering studies [1]. However, ripples produced by a plow just above onset always travel in the reverse direction, i.e. opposite to the direction of driving, as shown in Fig. 4. The backward travel reverses at higher driving speeds, but there is no smooth transition from backward to forward ripples; there is no speed where the ripples are stationary. If the speed is rapidly stepped from below to well above $v_{c}$, as in Fig. $3 \mathrm{p}$, the threshold speed above which backward ripples are observed in the steady state appears to scale in the same way as $v_{c}$, as shown in Fig. 10. Backward traveling ripples only exist for a narrow range of parameter values just above onset.

Another distinguishing feature of backward traveling ripples is that ripple merger events, which are common near onset for forward traveling ripples, generally do not occur. This is because the backward ripples appear at the same wavelength as in their amplitude-saturated state, 
whereas there is a strong relationship between the wavelength and amplitude for the forward ripples. This is related to another difference: that the plow does not leave the granular surface during backward ripple motion, while such ballistic motion is always present for forward traveling ripples using the plow.

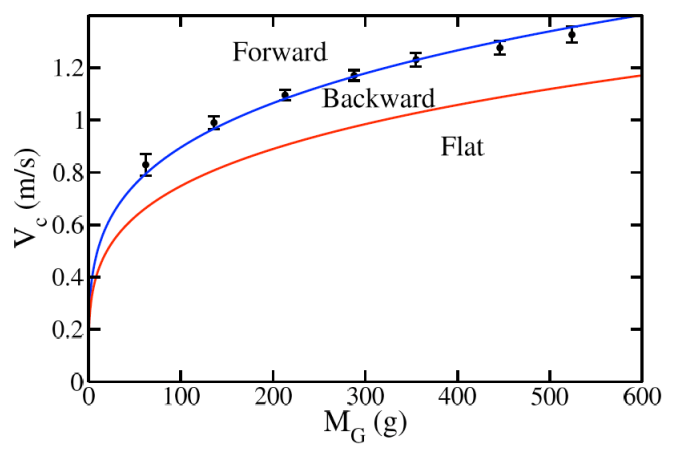

FIG. 10: (Color online) The domain of existence and transitions for forward and backward traveling ripples using the plow. The data and upper solid line shows the transition between backward and forward traveling ripples at $\mathrm{Fr}=3.80$. The lower solid line shows the onset of backward ripples from the flat bed at $\mathrm{Fr}=2.58$, as in Fig. 5

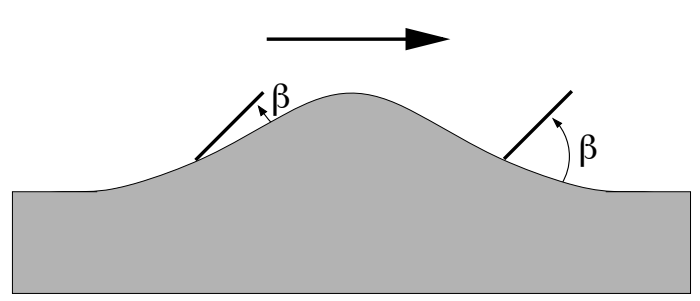

FIG. 11: Schematic mechanism for the formation of backward traveling ripples. The arrow shows the direction of motion of the plow. If the rate of erosion by the plow increases with the angle $\beta$ with the surface, then material is preferentially removed from the downward face of the ripple and deposited on the upward face of the following one, giving rise to a net backward ripple motion. During this process, the plow does not leave the surface.

The formation of backward traveling ripples is fundamentally different from the inertial mechanism of forward traveling ripples. Ripples can move backward even though the sand is only transported forward. The surface flux of grains in the driving direction depends not only on the normal force, but also on the geometry of the contact region. It is plausible to suppose that the surface flux increases with the angle $\beta$ between the plow and the granular surface. Initially, $\beta=\alpha$, the fixed angle of attack of the plow, but as the ripples develop, $\beta$ is modulated by the ripples as the plow remains in contact with the surface, as shown in Fig. 11. The result of this modulation is that $\beta$, and hence the surface flux, is smaller on the upward face of the ripple than on the downward one. The upward face is therefore less eroded, while the sand that is preferentially eroded from the downward face is deposited on the upward face of the following ripple. Thus, the net motion of the ripple is backward, while the grains are still transported forward. A similar mechanism has been proposed for the uphill motion of moguls on ski slopes [23].

Conversely, in the case of forward traveling ripples, inertial effects dominate and the plow leaves the surface and goes into ballistic motion near the crest of the ripple. The erosion is therefore all on the upward face, while the downward one forms a slip face which is added to by small avalanches consisting of material transported from the upward face. Thus, the ripples in the ballistic regime move forward, in the driving direction.

\section{MORE COMPLEX STATES}

In the preceding sections, we have focused on the scaling of the critical speed $v_{c}$ for the onset of the ripple pattern. While a complete study of the states above onset is beyond the scope of this paper, it is interesting to survey some of the complex states that are encountered above $v_{c}$. Fig. 12 shows a selection of such fully developed ripple states.

Other than the flat bed, the simplest states are pure traveling ripples, which are well described by single Fourier modes. For wheels, these always travel forward, while for the plow they travel backward near onset, as in Fig. $12 \mathrm{a}$, and forward for larger speeds, as in Fig. 12. As the driving speed $v$ of the plow is increased, the backward traveling ripple speed decreases, but is never zero. Increasing $v$ results in a low-dimensional chaotic state, as in Fig. $12 \mathrm{~b}$, in which several Fourier modes are excited. Further increasing $v$, we enter a regime of steady forward traveling ripples, but here the plow spends much of the time in a free, ballistic trajectory between impacts. If these impacts are not periodic around the table, there is some jitter between the phase of the ripples and that of the table; this is visible in Fig. 12k. At still higher speeds of the plow, a second chaotic regime is reached, as shown in Fig. 12 ,

The phenomenology for wheels differs in detail, but is similarly complex. For example, Fig. 12 and $f$ show wheel-driven ripple states consisting of two significant Fourier modes which are not simply related. The two ripple modes propagate with different speeds, producing a beating effect which persists indefinitely. All of these complex states for plows and wheels are reminiscent of the multiple dynamical states of impact oscillators 20 in their strongly nonlinear regime. 

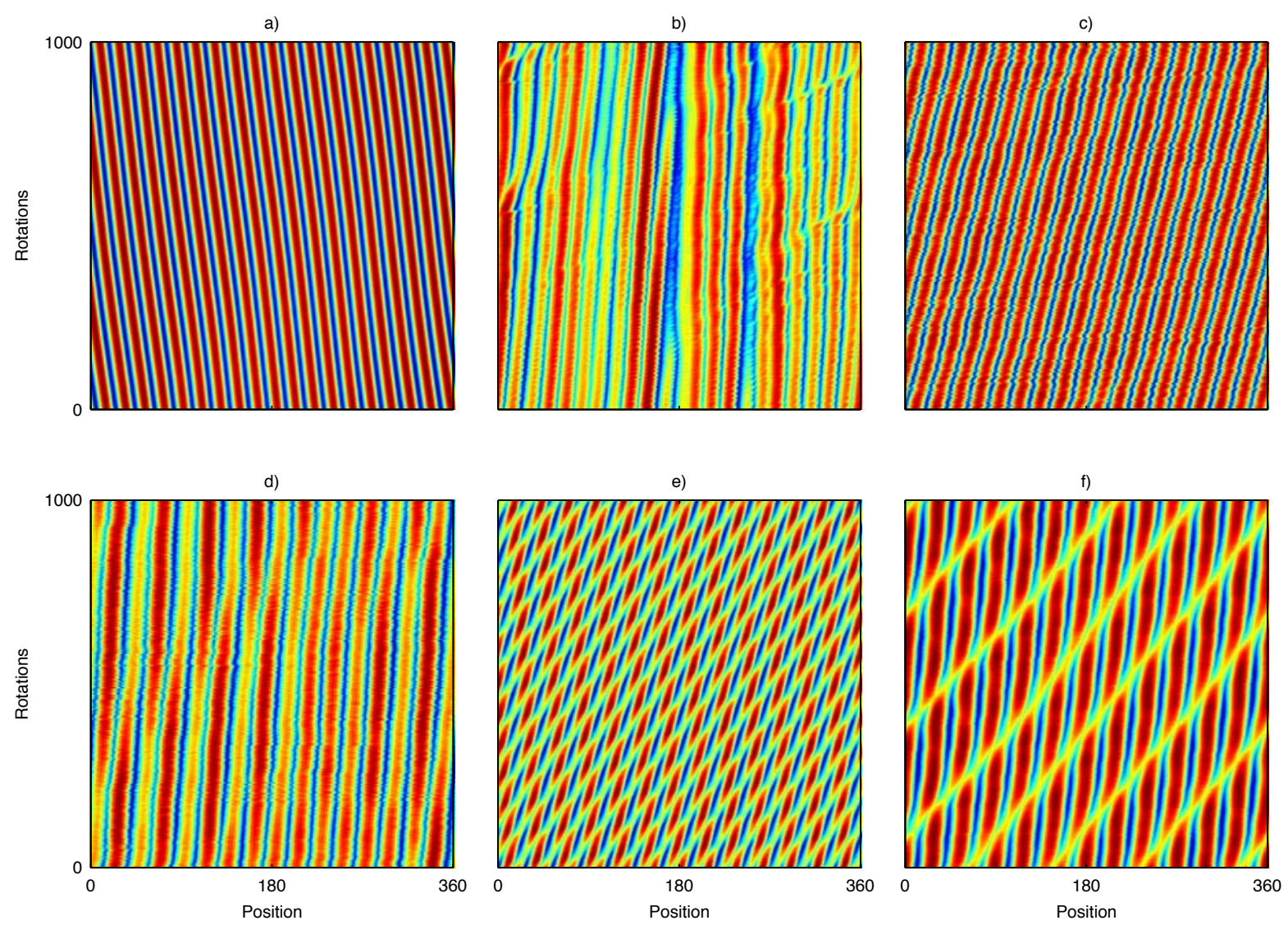

FIG. 12: The zoo of states for the plow and wheel. Each figure contains 1000 revolutions at constant speed. The range of ripple heights is normalized for each figure with red representing higher a) Plow at $0.737 \mathrm{~ms}^{-1}$. b) Plow at $0.827 \mathrm{~ms}^{-1}$. c) Plow at $0.964 \mathrm{~m} \mathrm{~s}^{-1}$. d) Plow at $1.001 \mathrm{~m} \mathrm{~s}^{-1}$. e) Wheel at $1.166 \mathrm{~m} \mathrm{~s}^{-1}$. f) Wheel at $1.325 \mathrm{~m} \mathrm{~s}^{-1}$

\section{CONCLUSION}

We have studied the rippling instability of a flat granular surface under the action of a moving source of stress. We considered both the classic case of a rolling wheel producing "washboard road" and the simpler case of a flat plow blade. In both cases, we focused on the region near the onset speed $v_{c}$. We developed a scaling theory for $v_{c}$ based on the idea that the onset of the instability represents a crossover between conservative, dynamic forces proportional to $v^{2}$ and dissipative, static forces which are independent of $v$. We found that $v_{c}$ did scale with the gravitational mass as $M_{G}$ as predicted for the plow and for non-rolling wheels. These have different scalings due to differences in their shape. However, the predicted mass scaling was not found for rolling wheels, suggesting that a more detailed theory of the surface penetration of a rolling wheel is required. We then considered the dependence of $v_{c}$ on various other geometric parameters.

Finally, we surveyed some of the complex ripple dynamics that are observed above onset, which include both forward and backward traveling ripples, chaotic states and states with traveling defects. Clearly, understanding the above-onset regime of finite amplitude ripples and their interactions will require a fully nonlinear theory of the instability. Such a theory would need to go well beyond the simple scaling of the onset speed $v_{c}$ that we have discussed here.

Throughout this work, we have used the angular position of the arm to trace the motion of the wheel or plow. To completely close the feedback loop between the moving source of stress and the state of the granular bed, we would also need to measure the shape of the granular surface and the rotational state of the wheel. This would allow us to determine the full acceleration of the wheel or plow, and hence the forces on it, as well as the dynamic response of the granular bed. The bed shape could be determined, for example, by a scanning laser profilometer 24. Another, somewhat complementary avenue to the same kind of information is soft sphere molecular dynamics simulation 8 in $2 \mathrm{D}$ or $3 \mathrm{D}$. With this data, a realistic dynamical model of the wheel-bed interaction could be developed, leading to a more complete continuum theory of the washboard instability. 


\section{Acknowledgments}

This work was supported by the George Batchelor Laboratory, University of Cambridge, the Swiss National Science Foundation, the Natural Science and Engineering
Research Council (NSERC) of Canada, the Isaac Newton Trust and the Engineering and Physical Sciences Research Council (UK). Many thanks to Stuart Dalziel, director of the Batchelor laboratory.
[1] W. Heath and R. Robinson, Transport and Road Research Laboratory, Suppl. Report No. 610 (1980).

[2] K. B. Mather, Civ. Eng. Pub. Works Rev., 57, 617 (1963), and Civ. Eng. Pub. Works Rev., 57, 781 (1963).

[3] S. Shoop, R. Haehnel, V. Janoo, D. Harjes and R. Liston, J. Geotech. Geoenviron. Eng., 132, 852 (2006).

[4] J. G. Riley and R. B. Furry, Highway Research Record, 438, 54 (1973).

[5] J. G. Riley, Ph.D thesis, Cornell University (1971).

[6] R. W. Grau and L. B. Della-Moretta, Trans. Res. Rec. 1291, Vol. 2, 313 (1991).

[7] G. K. Misoi and R. M. Carson, Proc. Instn. Mech. Engrs., 203, 205 (1989), and Proc. Instn. Mech. Engrs., 203, 215 (1989).

[8] N. Taberlet, S. W. Morris and J. N. McElwaine, Phys. Rev. Let., 99, 068003 (2007).

[9] M. C. Cross and H. Greenside, Pattern Formation and Dynamics in Nonequilibrium Systems, Cambridge University Press, 2009.

[10] D. C. Mays and B. A. Faybishenko, Complexity, 5, 51 (2000).

[11] J. A. Both, D. C. Hong and D. A. Kurtze, Physica A, 301, 545 (2001).

[12] R. A. Bagnold, The Physics of Blown Sand and Desert Dunes, Methuen, London 1941.

[13] F. Engelund and J. Fredsoe, Ann. Rev. Fluid Mech., 14,
$13(1982)$

[14] Y. Sato, A. Matsumoto and K. Knothe, Wear, 253, 130 (2002).

[15] J. Egger, Physica D, 165, 127 (2002).

[16] C. Clanet, F. Hersen and L. Bocquet, Nature, 427, 29 (2004).

[17] Q. Dai, F. Hendriks and B. Marchon, J. Appl. Phys., 96 696 (2004).

[18] R. Pit, B. Marchon, S. Meeks and V. Velidandla, J. Trib. Lett., 10133 (2001).

[19] W. Chin, E. Ott, H. E. Nusse and C. Grebogi, Phys. Rev. E, 504427 (1994).

[20] S. R. Bishop, Phil. Trans. R. Soc. Lond. A, 347, 347 (1994).

[21] POSIROT from ASM limited. Part No: PRAS1-30-U2CW-M12 modified to operate at $2 \mathrm{kHz}$.

[22] The hydrodynamic Froude number is given either by $v^{2} / g L$, or sometimes by the square root of this quantity. It is usually physically explained as being the ratio of a speed $v$ and the wave speed of a surface gravity wave of wavelength $L$, all squared. Of course, no gravity waves are involved in the formation of the washboard ripple pattern.

[23] David Bahr, private communication. [Link].

[24] LLT2800-100 laser triangulator from Micro-Epsilon Ltd. 